Revue de droit comparé du travail et de la sécurité sociale

3| 2018

La participation des travailleurs dans la grande entreprise privée et publique

\title{
La prohibition des discriminations raciales sur le lieu de travail
}

Dominique Allen

\section{CpenEdition}

Journals

Édition électronique

URL : https://journals.openedition.org/rdctss/1925

DOI : $10.4000 /$ rdctss. 1925

ISSN : 2262-9815

Éditeur

Centre de droit comparé du travail et de la sécurité sociale

Édition imprimée

Date de publication : 1 novembre 2018

Pagination : 156-159

ISSN : 2117-4350

Référence électronique

Dominique Allen, « La prohibition des discriminations raciales sur le lieu de travail », Revue de droit comparé du travail et de la sécurité sociale [En ligne], 3 | 2018, mis en ligne le 01 novembre 2021, consulté le 13 novembre 2021. URL : http://journals.openedition.org/rdctss/1925 ; DOI : https:// doi.org/10.4000/rdctss. 1925

\section{(c) (i) (9)}

Revue de droit comparé du travail et de la sécurité sociale est mise à disposition selon les termes de la Licence Creative Commons Attribution - Pas d'Utilisation Commerciale - Pas de Modification 4.0 International. 


\section{DOMINIQUE ALLEN}

UNIVERSITÉ DE MONASH

\section{LA PROHIBITION DES DISCRIMINATIONS RACIALES SUR LE LIEU DE TRAVAIL}

Depuis plus de quarante ans, en Australie, la discrimination sur le lieu de travail est interdite. Jusqu'à récemment, la loi contre les discriminations s'appliquait uniquement à cette question : la loi sur les relations professionnelles interdisait le licenciement uniquement basé sur un motif prohibé, comme le sexe ou la race. La loi de 2009 sur le travail équitable (Fair Work Act 2009 Commonwealth, "FW Act ») a étendu la loi sur les relations professionnelles à d'autres formes de discrimination, et a donné au Médiateur pour le travail équitable (Fair Work Ombudsman, "FWO») le pouvoir de faire respecter la loi, ce que les organismes créés en vertu des lois anti-discrimination n'ont jamais été en mesure de faire.

Au début, le FWO était très actif dans ce domaine, mais il s'est ensuite concentré sur d'autres infractions à la loi FW. Récemment, il a obtenu gain de cause dans une affaire de discrimination fondée sur la race et la nationalité, qui a également été la première plainte de discrimination raciale déposée en vertu de la loi FW. Dans l'affaire Fair Work Ombudsman vs Yenida Pty Ltd ( "Yenida »'), le tribunal a décidé qu'un hôtel avait discriminé deux salariés étrangers en les payant moins et en les traitant moins bien que les salariés australiens. Dans cette contribution, nous comparons le traitement des plaintes de discrimination sur le lieu de travail dans le cadre de la législation anti-discrimination à celle des relations professionnelles et montrons que le fait de disposer d'un organisme officiel doté de pouvoirs variés pour appliquer cette loi pourrait changer la donne en matière de discriminations sur le lieu de travail en Australie. La dernière partie est consacrée à l'affaire Yenida.

\section{I - FAIRE VALOIR LES PLAINTES DE DISCRIMINATION SELON LA LOI ANTI-DISCRIMINATION}

En 1975, le gouvernement fédéral australien a adopté la première loi anti-discrimination du pays, la loi sur la discrimination raciale (Racial Discrimination Act, Commonwealth). Peu après, les États et territoires ont commencé à promulguer des lois interdisant la discrimination fondée sur le genre, suivis d'autres motifs. La discrimination au travail est maintenant interdite dans tout le pays pour divers motifs ${ }^{2}$. Les lois sont très semblables, surtout en ce qui concerne leur application. La personne victime de discrimination doit déposer plainte auprès de l'Agence pour l'égalité des chances de sa juridiction ou auprès de la Commission australienne des droits de l'Homme. Il est impossible de saisir directement les tribunaux, sauf à Victoria ${ }^{3}$. Sous réserve que la plainte soit fondée et qu'elle soit du ressort de l'organisme, celui-ci tentera de la régler au moyen d'un mode alternatif

1 [2017] FCCA 2299.

2 Voir également N. Rees, S. Rice, D. Allen, Australian Anti-Discrimination and Equal Opportunity Law, The Federation Press, 2018.

3 Loi de 2010 sur l'égalité des chances (Vic) Partie 8. 


\section{Australie}

de règlement des conflits ( MARC »), qui est un processus confidentiel. La plupart des plaintes sont réglées ou retirées. Peu d'entre elles sont entendues par un tribunal civil ou, dans le cas de plaintes au niveau fédéral, par les tribunaux fédéraux. Si ces plaintes sont portées devant les tribunaux, elles font l'objet d'un deuxième MARC et bon nombre d'entre elles sont alors réglées. Les tribunaux ne traitent que très peu de plaintes chaque année. Cela s'explique notamment par les coûts financiers, psychologiques et émotionnels liés au litige, par le fait que le salarié est satisfait de la conclusion du litige et par le délai entre la survenance de la discrimination et la date prévue pour la comparution devant le tribunal. Autre facteur influant sur le règlement : les dommages-intérêts accordés dans les affaires de discrimination se sont souvent révélés faibles ${ }^{4}$.

Contrairement à de nombreux autres pays, les agences pour l'égalité des chances ne disposent d'aucune marge de manœuvre pour faire appliquer la loi, que ce soit en apportant une aide financière à un salarié ou en se saisissant du dossier pour lui. Compte tenu de leur rôle dans la réception des plaintes et la mise en œuvre du MARC, il y aurait un risque de conflit d'intérêts si les agences pour l'égalité des chances se voyaient octroyer des pouvoirs d'exécution, leur rôle principal étant donc le traitement des plaintes et la mise en $œ u v r e$ de MARC. Elles sont également chargées de faire connaître la loi à la communauté et de veiller à son respect.

\section{II - FAIRE VALOIR LES PLAINTES DE DISCRIMINATION DANS LA LOI SUR LE TRAVAIL ÉQUITABLE}

L'introduction de la loi FW a changé la donne en étendant l'interdiction de la discrimination par-delà du licenciement et en créant un organe chargé de faire respecter la loi.

L'article 351 de la loi FW interdit à un employeur de prendre des "mesures défavorables » contre un salarié en raison de sa race, de son sexe, de son âge ou de son handicap, par exemple. Un comportement contraire à la loi, c'est-à-dire le fait d'adopter une «mesure défavorable ", comprend : le licenciement du salarié, le préjudice causé à son encontre dans son emploi, la rétrogradation de son poste et la discrimination à son égard. Toute menace ou action de ce type est interdite. Les salariés actuels et futurs sont protégés. Une fois établie la preuve de la violation de l'article 351, le renversement de la charge de la preuve incombe à l'employeur qui peut alors renverser la présomption.

L'application de la loi FW ne repose pas sur un individu. Les syndicats peuvent déposer une plainte au nom de leurs membres. Plus important encore, l'organisme officiel fédéral, le FWO, a le pouvoir d'enquêter sur les manquements à la loi et de la faire respecter. Les inspecteurs chargés du travail équitable peuvent mener une enquête sur une infraction présumée et, s'ils ont des motifs raisonnables de croire que la loi a été enfreinte, le FWO peut alors accepter un engagement exécutoire de l'employeur. Par exemple, un commerçant a accepté de suivre une formation, de publier un avis reconnaissant son comportement illicite dans un journal et de l'afficher sur le lieu de travail, une enquête ayant révélé qu'il était coupable de discrimination à l'égard d'une salariée enceinte, en réduisant ses heures de travail et en donnant du travail à de nouveaux salariés plutôt qu'à elle. L'employeur

4 Voir également D. Allen, «Behind the Conciliation Doors : Settlement Discrimination Complaints in Victoria », Griffith Law Review, 2009, n²18, p. 78. 
a également accepté d'indemniser la salariée et de lui remettre des excuses écrites ${ }^{5}$. Si l'employeur n'est pas disposé à s'y conformer de son plein gré, le FWO peut intenter une action en justice et demander une indemnisation pour le salarié concerné et une amende administrative qui peut atteindre 60 unités par infraction pour une personne physique (actuellement 12600 AUD \$) et cinq fois ce montant pour une personne moraleb.

\section{III - FAIR WORK OMBUDSMAN VS YENIDA PTY LTD}

L'affaire Yenida a associé deux des domaines actuellement prioritaires parmi les activités de conformité du FWO : les travailleurs migrants et le secteur hôtelier. Le FWO constate d'ailleurs que sa charge de travail s'est excessivement alourdie par le nombre de détenteurs de visas et que bon nombre d'entre eux sont de jeunes travailleurs du secteur hôtelier ${ }^{7}$. L'industrie hôtelière représente près d'un tiers de la charge de travail du FWO en matière de litiges, alors que le personnel hôtelier représente une très faible part du marché du travail australien ${ }^{8}$. Les contrôles réalisés auprès des entreprises de ce secteur ont souvent conduit le FWO à intervenir pour réclamer les salaires impayés. En effet, même si dans l'affaire Yenida', il était question de salaires insuffisants, l'objet du litige était surtout la façon dont l'employeur a traité deux travailleurs étrangers par rapport à ses employés australiens.

L'affaire mettait en cause un hôtel en Tasmanie appartenant à Yenida et géré par l'un de ses directeurs, M. Chang. M. Loh y travaillait comme chef cuisinier et son épouse, Mme Low, comme aide de cuisine. Tous deux étaient ressortissants malaisiens d'origine chinoise. Ils ont quitté la Malaisie suite à une offre d'emploi publiée par M. Chang dans un journal malaisien, à laquelle $M$. Loh a répondu. Le couple a vécu sur place et a travaillé dans l'hôtel pendant près de sept ans. M. Chang a sponsorisé le visa de travail de M. Loh et le visa de Mme Low dépendait de celui de son mari.

Le FWO a reproché à Yenida et à $\mathrm{M}$. Chang d'avoir discriminé le couple en raison de leur race et de leur nationalité. M. Loh devait travailler six jours par semaine, il n'avait pas droit ni aux congés ni aux jours fériés et il recevait toujours le même salaire, quel que soit le nombre d'heures effectuées. En comparaison le chef cuisinier né en Australie, travaillait moins d'heures et elles étaient réparties sur cinq jours. Comme son mari, le salaire hebdomadaire de Mme Low ne variait pas en fonction de ses heures de travail.

Le terme "discrimination » n'est pas défini dans la loi FW; on lui a attribué le sens $d^{\prime} u n$ " traitement moins favorable ${ }^{10}$. Dans l'affaire Yenida, le juge Baker a conclu que l'employeur avait traité M. Loh de façon moins favorable en omettant de lui verser des taux majorés et en n'exigeant pas de sa part d'inscrire sur une feuille de temps toutes ses heures travaillées. L'employeur a aussi fait preuve de discrimination à l'égard de Mme Low en lui

5 Engagement juridiquement contraignant Shawna Pty Ltd (29 janvier 2013). https://www.fairwork.gov.au/ArticleDocuments/721/Redacted-Shawna-EU-English.pdf.aspx

6 FW Act ss 539 Item 11, 546.

7 FWO, Annual Report 2016-17, 2017, 18-19.

8 Ibid, p. 20-21. Voir aussi M. Bailey, «Fair Work Ombudsman wins first racial discrimination case», Australian Financial Review, 27 May 2018.

9 Le FWO reprochait à l'hôtel d'avoir sous-payé 15 salariés et d'avoir omis de tenir des registres, ce que l'employeur a admis au moment où la plainte a été examinée par le tribunal. Tous ces salariés étaient des citoyens australiens d'origine caucasienne : Yenida [11].

10 Hodkinson vs Commonwealth (2011) 248 FLR 409. 


\section{Australie}

versant un salaire hebdomadaire fixe qui ne variait pas en fonction du nombre d'heures travaillées.

$\mathrm{Ni} \mathrm{la} \mathrm{«} \mathrm{race} \mathrm{»} \mathrm{ni} \mathrm{la} \mathrm{«nationalité} \mathrm{»} \mathrm{ne} \mathrm{sont} \mathrm{définies} \mathrm{dans} \mathrm{la} \mathrm{loi} \mathrm{FW.} \mathrm{Le} \mathrm{juge} \mathrm{Baker} \mathrm{a} \mathrm{repris}$ le sens ordinaire des deux termes tels qu'ils figurent dans le dictionnaire et a conclu que la race du couple était chinoise et que leur nationalité était malaise. M. Chang n'a pas été en mesure de démontrer que la race et/ou la nationalité du couple ne constituaient pas un motif solide et pertinent pour lequel il les a traités de façon moins favorable. Le juge Baker a considéré qu'il avait recruté $M$. Loh parce qu'il se doutait qu'un Malaisien accepterait probablement de travailler six jours par semaine, chose courante dans ce pays.

Non seulement le juge Baker a conclu que l'employeur avait agi illégalement, mais aussi que M. Chang était responsable de toutes ces contraventions. Au moment où l'affaire a été entendue, M. Chang avait déjà vendu l'hôtel parce qu'il n'était pas très rentable. Lorsque la décision sur les pénalités a été entendue début 2018, M. Chang a déclaré qu'il avait dû emprunter de l'argent pour rembourser le salaire de ses anciens salariés et il a affirmé ne pas pouvoir payer de pénalités ${ }^{11}$. Toutefois, pour déterminer la pénalité appropriée, le juge Baker a précisé qu'il fallait accorder plus d'importance à la dissuasion qu'à la capacité de payer de l'employeur ${ }^{12}$. Son Honneur a imposé des pénalités à Yenida et à M. Chang pour avoir enfreint les dispositions de l'article 351. Bien que ces montants soient très faibles, des pénalités ont également été appliquées pour la plupart des autres infractions à la loi FW (à savoir, sous-payer les autres salariés). Ces pénalités ont été chiffrées à 176005 \$ pour Yenida et 35099 \$ pour M. Chang, en plus des 52 017,74 \$ de salaires impayés.

\section{Conclusion}

Le FWO a été le premier à se lancer dans l'application des lois sur la discrimination raciale et l'affaire Yenida s'avère être un véritable succès. Comme il s'agit pour le FWO de la première affaire de discrimination en trois ans, Yenida a également permis de mobiliser l'attention sur cette question, plutôt que de laisser à chaque salarié le soin de faire respecter la loi. Il est important pour le FWO de maintenir son rôle dans ce domaine pour deux raisons. Premièrement, la jurisprudence concernant l'article 351 étant sous-développée, il serait avantageux pour tous les salariés que le FWO se charge des affaires et élabore sa jurisprudence de façon stratégique. Deuxièmement, le FWO est le seul organe habilité à faire appliquer les lois anti-discrimination et doit donc continuer à encourager leur respect en menant des enquêtes et en intentant des poursuites si nécessaire. Cela dit, il faut trouver un équilibre entre le fait d'encourager le respect de la loi par des poursuites judiciaires et le fait d'agir dans l'ombre des litiges. Dans une affaire récente de salaires insuffisants, le tribunal s'est montré très critique à l'égard de la stratégie du FWO qui consiste à intenter une poursuite contre un restaurant. En imposant la pénalité minimale, le juge McNab a tenu compte de l'impact de la " publicité négative » que le restaurateur et ses établissements avaient subie à la suite des mesures d'exécution prises par le FWO et a déclaré que, dans ce cas, des engagements exécutoires auraient pu être envisagés à la place ${ }^{13}$. Le choix de la ligne de conduite la plus appropriée varie en fonction de la nature de l'infraction.

11 Fair Work Ombudsman vs Yenida Pty Ltd [2018] FCCA 1342, [104]-[111].

12 Ibid [126].

13 Fair Work Ombudsman vs The Meatball And Wine Bar Pty Ltd [2018] FCCA 2288. 